\title{
Transpedal Approach for the Treatment of Acute Limb Ischemia in a Patient with Limited Access Sites
}

\author{
Robert Abed, MD, MPH ${ }^{1 *}$, Naveed Rajper, $M D^{2}$, Joseph Puma, $M D^{2}$ and Justin Ratcliffe, $M D^{2}$ \\ ${ }^{1}$ Internal Medicine Resident, Mount Sinai St. Luke's and West, USA \\ ${ }^{2}$ Interventional Cardiology, Mount Sinai St. Luke's, USA \\ *Corresponding author: Robert Abed, MD, MPH, Internal Medicine Resident, Mount Sinai St. Luke's and \\ West 10 Amsterdam Avenue, Apt 1003, New York, NY, 10023, USA, Tel: +1-(929)-284-5178
}

\begin{abstract}
We present a 53-year-old male with a history of extensive peripheral vascular disease, including a repaired type B aortic dissection complicated by occlusion of the left subclavian artery requiring carotid to subclavian bypass. The patient then had a subsequent repair of a graft endoleak complicated by left iliac artery occlusion requiring fem-fem bypass. He presented to the ED with left foot pain concerning for acute limb ischemia needing for prompt revascularization. However, given the extensive prior vascular surgeries, traditional femoral approaches were unable to be utilized. Therefore, a retrograde ipsilateral transpedal approach was chosen as the only remaining possible access site for angiography and intervention. The left anterior tibial artery was cannulated via a 4 French access. The angiogram revealed a total occlusion in the left posterior tibial artery with poor pedal arch flow. Via the 4Fr system, treatment with mechanical thrombectomy, percutaneous transluminal angioplasty, and thrombolysis with tPA was performed and successful restoration of flow to the limb was achieved.
\end{abstract}

\section{Keywords}

Acute limb ischemia, Pedal approach, Thrombolysis, Percutaneous transluminal angioplasty, Thrombectomy

\section{Learning Objective}

Peripheral artery disease (PAD) is growing into a worldwide pandemic with a prevalence as high as $12 \%$ in developed countries [1]. Acute limb ischemia (ALI), which is the most severe form of PAD, is described as a rapid onset decrease of limb perfusion that threatens limb viability with almost all cases being related to arterial occlusion [2]. While ALI constitutes only $1-3 \%$ of symptomatic PAD, it has become more prevalent as a result of aging populations, and rising rates of diabetes and chronic kidney disease (CKD). The cornerstone of limb salvage remains early revascularization combined with maximal optimal medical therapy and wound care. However, despite multiple advances, the management of this disease remains a challenge which is reflected in prevailing high rates of mortality. Research has shown that $20 \%$ of patients will die within one year after being diagnosed with ALI [3]. Current advances in endovascular therapy enable prompt treatment of ALI however, most treatment has been performed via traditional femoral artery access sites.

The case aims to illustrate the feasibility of a retrograde ipsilateral transpedal approach when the commonly used contralateral femoral and ipsilateral anterograde femoral approaches are inaccessible for the treatment of distal lower limb ALI.

\section{Background of the Patient}

We present a 53-year-old male with an extensive smoking history and a past medical history significant for hypertension, chronic kidney disease stage (CKD) $\mathrm{G} 3$, and heart failure with preserved ejection fraction. Also, he has an extensive history of peripheral arterial disease (PAD). He was previously diagnosed with a type $B$ aortic dissection complicated with sac expansion and underwent thoracic endovascular aortic repair and endovascular aneurysm repair (TEVAR/EVAR) complicated by occlusion of the left carotid and subclavian arteries

Citation: Abed R, Rajper N, Puma J, Ratcliffe J (2020) Transpedal Approach for the Treatment of Acute Limb Ischemia in a Patient with Limited Access Sites. Int J Clin Cardiol 7:186. doi.org/10.23937/23782951/1410186

Accepted: June 25, 2020; Published: June 27, 2020

Copyright: (C) 2020 Abed R, et al. This is an open-access article distributed under the terms of the Creative Commons Attribution License, which permits unrestricted use, distribution, and reproduction in any medium, provided the original author and source are credited. 


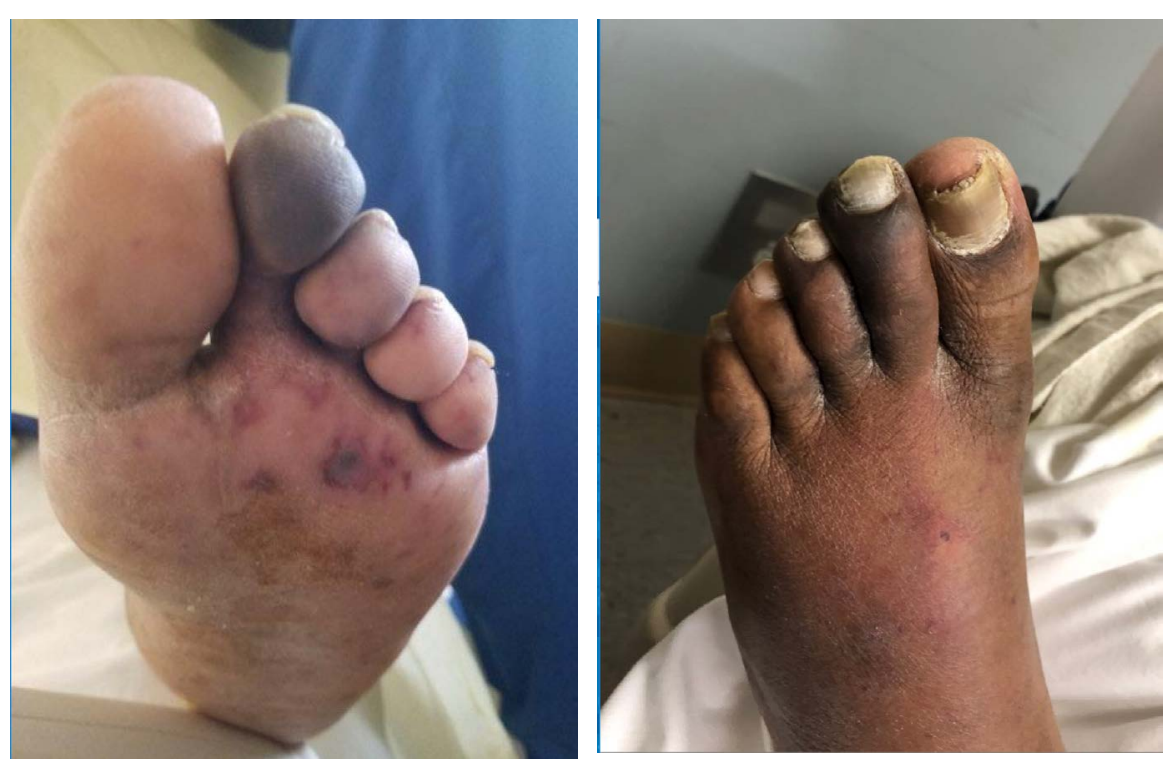

Figure 1: Ischemic foot.

requiring right carotid to carotid and carotid to left subclavian bypass. One month later, he underwent surgery for a type II endoleak complicated by left iliac artery occlusion requiring a fem-fem bypass. The patient was discharged and was stable and very functional for the past five years until the current presentation of acute symptoms.

His home medications include amlodipine, clopidogrel, disopyramide, furosemide, labetalol, losartan and minoxidil.

\section{Clinical Presentation}

Our patient presented to the emergency department with a chief complaint of left foot pain. Before this visit, the patient was in his usual state of health. He reports a 5-day history of progressively increasing left leg and foot pain radiating to the distal toes, associated with worsening pallor and weakness. The review of systems was noncontributory. His vital signs were unremarkable. On physical examination, in comparison to the right limb, his left dorsalis pedis and posterior tibial pulses were not audible on doppler assessment, his toes appeared mottled, blue, cold and tender to touch. On neurological examination of the lower extremities, there was a notable decrease in sensation and a moderate weakness in the left foot compared to the right. The rest of the exam was noncontributory (Figure 1).

\section{Diagnostic Focus and Assessment}

Given the extensive PAD history, there was high suspicion for acute limb ischemia (ALI Stage IIb) and the vascular team was consulted, and an urgent angiogram was planned. The team faced a challenge in choosing an adequate vascular access site in a patient with an extensive surgical bypass history in both his lower and upper extremities. Both contralateral femoral and ipsilateral anterograde femoral accesses were not an option. Therefore, a retrograde ipsilateral transpedal ap- proach was chosen as it was the only remaining option. Ultrasound-guided access of the anterior tibial (AT) artery was performed and a 4Fr Pinnacle precision sheath (Terumo) was placed. Subsequent angiography through a Vertebral catheter (Cordis) revealed a patent fem-fem bypass, chronic total occlusion of the left iliac artery, patent superficial femoral artery (SFA) and popliteal arteries and total occlusion in the left posterior tibial (PT) artery with a reduced flow into the foot and likely the embolic culprit to the patient's acute symptoms (Figure 2).

\section{Therapeutic Focus and Assessment}

In order to treat the thrombus in the PT artery from the AT arterial access site, an "up and over" approach was used. Using an IM catheter (Cordis) and a 0.014" Run through wire (Terumo), we were able to cross over from the AT artery into the PT artery then cannulate the distal total occlusion of the PT and wire into the pedal arch itself.

The embolic lesion was treated with mechanical thrombectomy (Penumbra using a CAT4 catheter) and balloon angioplasty with a $3.5 \times 150$ Saber (Cordis). Flow in the PT artery was restored, but significant thrombus burden was noted in the pedal arch, so $10 \mathrm{mg}$ of tPA was slowly perfused over 30 minutes.

A final angiogram showed restoration of flow in the pedal arch (Figure 3). A Vasostat closure device was placed over the AT access site, and the patient was transferred to the CCU for further monitoring.

\section{Discussion}

This case demonstrates the successful treatment of ALI via a retrograde ipsilateral transpedal approach, in contrast to the more commonly used contralateral femoral, or antegrade ipsilateral femoral approaches. In this specific case, transpedal access was the only avail- 


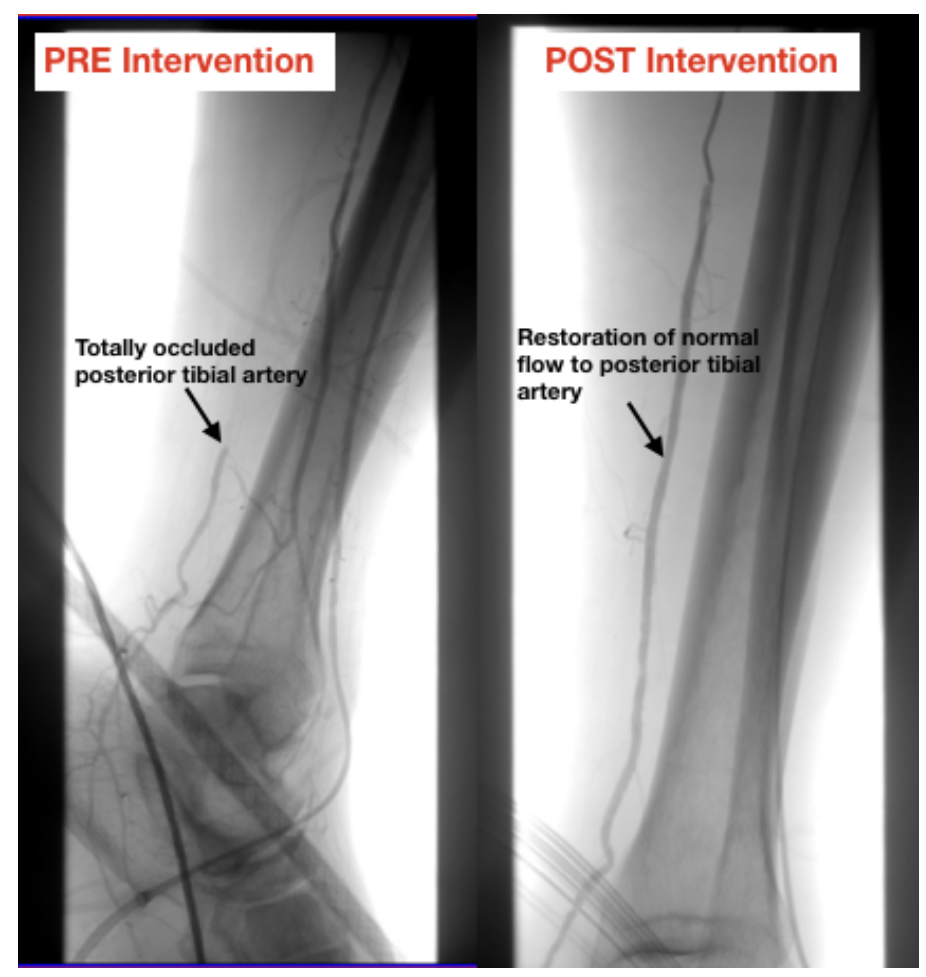

Figure 2: Pre- and post-intervention angiogram. Arrow points to posterior tibial artery, highlighting restoration of normal flow after intervention.

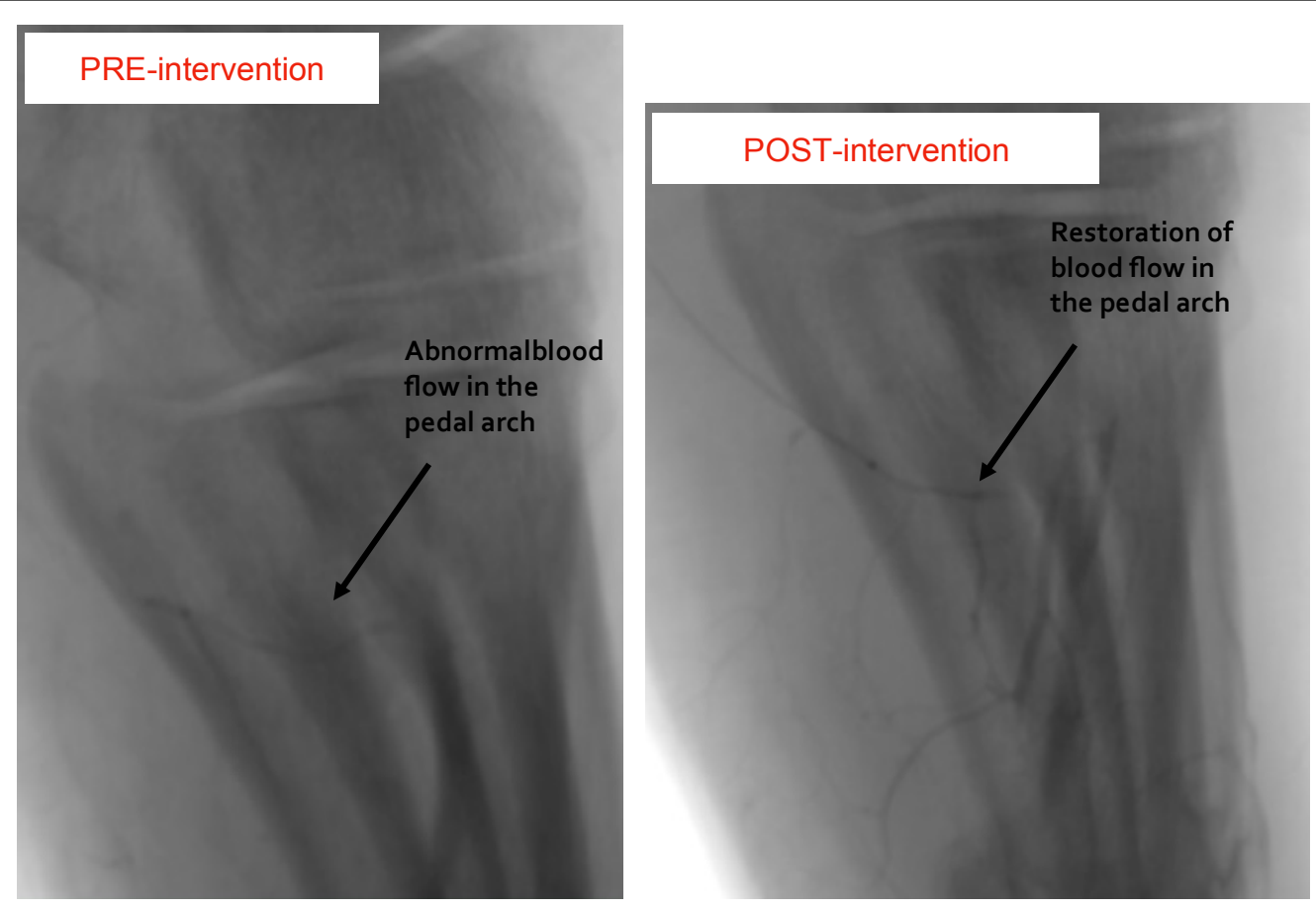

Figure 3: Pre- and post-intervention angiogram. Arrow points to pedal arch, highlighting restoration of normal flow after intervention.

able option for endovascular treatment. It is essential to highlight the variety of treatment options available through $4 \mathrm{Fr}$ transpedal access, including mechanical thrombectomy, angioplasty, and even the use of tPA in case a rare case like this patient arises.

Our patient presented with history and physical exam findings that were very concerning for ALI. He had a known extensive PAD history. It has been shown that $5-10 \%$ of patients with PAD will develop ALI in the next five years [3]. Also, most ALI patients have associated multilevel disease involving two or more territories, which our patient had in his past medical history [4]. Other risk factors associated with distal vessel involvement include older age (> 80), hypertension, diabetes, and CKD [5].

Once $A L I$ is suspected, patients generally undergo non-invasive testing (CTA, MRA) to establish a diagnosis. That being said, it is essential to highlight that these im- 
aging modalities are very limited when used to identify very distal disease [6]. In our case, given his extensive PAD, it was decided to proceed directly with an angiogram with the intention of endovascular revascularization as time was of the essence. As with any endovascular therapy, the procedure should be well planned out, starting with the access. For suprapopliteal lesions, vascular access may be obtained from the contralateral femoral access. However, for infrapopliteal lesions, such as tibial and pedal plantar interventions, contralateral femoral access is not as optimal because it requires a longer sheath and excessive contrast use. Those limitations can be overcome by an antegrade ipsilateral femoral access, usually at the level of the common femoral artery (CFA) for ease to apply compression and better torqueability, pushability and steerability. In our case, both of these approaches were not applicable given the extensive PAD history and vascular manipulation our patient had undergone in his past. There have been studies showing transpedal access for endovascular interventions. Ruzsa, et al. looked at transpedal access after failed anterograde recanalization of complex belowthe-knee and femoropopliteal occlusions in critical limb ischemia and found low acute and late complication rate [7]. Hence, it was decided that a retrograde ipsilateral pedal approach would be the best option.

Although this was not standard practice and further studies are necessary to promote further use of this technique, this experience suggests that a transpedal approach should be considered as a bailout strategy when other vascular sites are not possible to save a critical limb. Further studies should focus on assessing the efficacy and safety of transpedal vascular access for the management of acute limb ischemia.

\section{Conclusion}

In summary, this is a case of a patient who presented with $\mathrm{ALI}$ and was successfully treated with mechanical thrombectomy, PTA, and thrombolysis via a retrograde ipsilateral transpedal approach as traditional transfem- oral access sites were not available due to prior bypass surgery.

\section{Conflict of Interest}

None of the authors involved in this case have a conflict of interest.

\section{Declarations}

- Consent to write this case from patient was obtained.

- We do not report any competing interests.

- This study was not funded.

- Authors collaborated together on all aspects of the projects, including design, statistical analysis, manuscript writing.

\section{References}

1. Criqui $\mathrm{MH}$, Aboyans $\mathrm{V}$ (2015) Epidemiology of peripheral artery disease. Circ Res 116: 1509-1526.

2. Norgren L, Hiatt WR, Dormandy JA, Nehler MR, Harris KA, et al. (2007) Inter-society consensus for the management of peripheral arterial disease (TASC II). Eur J Vasc Endovasc Surg 33: S1-S75.

3. Varu VN, Hogg ME, Kibbe MR (2010) Critical limb ischemia. J Vasc Surg 51: 230-241.

4. Faglia E, Favales F, Quarantiello A, Calia P, Clelia P, et al. (1998) Angiographic evaluation of peripheral arterial occlusive disease and its role as a prognostic determinant for major amputation in diabetic subjects with foot ulcers. Diabetes Care 21: 625-630.

5. Aboyans V, Desormais I, Lacroix $P$, Salazar J, Criqui $M H$, et al. (2010) The general prognosis of patients with peripheral arterial disease differs according to the disease localization. J Am Coll Cardiol 55: 898-903.

6. Ndip A, Jude EB (2009) Emerging evidence for neuroischemic diabetic foot ulcers: Model of care and how to adapt practice. Int J Low Extrem Wounds 8: 82-94.

7. Ruzsa Z, Nemes B, Bánsághi Z, Tóth K, Kuti F, et al. (2013) Transpedal access after failed anterograde recanalization of complex below-the-knee and femoropoliteal occlusions in critical limb ischemia. Catheter Cardiovasc Interv 83: 997-1007. 